UDC 517.53

\author{
YA. I. SAVCHUK, A. I. BANDURA
}

\title{
ASYMPTOTIC VECTORS OF ENTIRE CURVES
}

Ya. I. Savchuk ${ }^{1}$, A. I. Bandura ${ }^{2}$. Asymptotic vectors of entire curves, Mat. Stud. 56 (2021), $48-54$.

We introduce a concept of asymptotic vector of an entire curve with linearly independent components and without common zeros and investigate a relationship between the asymptotic vectors and the Picard exceptional vectors.

A non-zero vector $\vec{a}=\left(a_{1}, a_{2}, \ldots, a_{p}\right) \in \mathbb{C}^{p}$ is called an asymptotic vector for the entire curve $\vec{G}(z)=\left(g_{1}(z), g_{2}(z), \ldots, g_{p}(z)\right)$ if there exists a continuous curve $L: \mathbb{R}_{+} \rightarrow \mathbb{C}$ given by an equation $z=z(t), 0 \leq t<\infty,|z(t)|<\infty, z(t) \rightarrow \infty$ as $t \rightarrow \infty$ such that

$$
\lim _{\substack{z \rightarrow \infty \\ z \in L}} \frac{\vec{G}(z) \vec{a}}{\|\vec{G}(z)\|}=\lim _{t \rightarrow \infty} \frac{\vec{G}(z(t)) \vec{a}}{\|\vec{G}(z(t))\|}=0
$$

where $\|\vec{G}(z)\|=\left(\left|g_{1}(z)\right|^{2}+\ldots+\left|g_{p}(z)\right|^{2}\right)^{1 / 2}, \vec{G}(z) \vec{a}=g_{1}(z) \cdot \bar{a}_{1}+g_{2}(z) \cdot \bar{a}_{2}+\ldots+g_{p}(z) \cdot \bar{a}_{p}$. A non-zero vector $\vec{a}=\left(a_{1}, a_{2}, \ldots, a_{p}\right) \in \mathbb{C}^{p}$ is called a Picard exceptional vector of an entire curve $\vec{G}(z)$ if the function $\vec{G}(z) \vec{a}$ has a finite number of zeros in $\{|z|<\infty\}$.

We prove that any Picard exceptional vector of transcendental entire curve with linearly independent components and without common zeros is an asymptotic vector. Here we demonstrate that the exceptional vectors in the sense of Borel or Nevanlina and, moreover, in the sense of Valiron do not have to be asymptotic. For this goal we use an example of meromorphic function of finite positive order, for which $\infty$ is no asymptotic value, but it is the Nevanlinna exceptional value. This function is constructed in known Goldberg and Ostrovskii's monograph "Value Distribution of Meromorphic Functions". Other our result describes sufficient conditions providing that some vectors are asymptotic for transcendental entire curve of finite order with linearly independent components and without common zeros. In this result, we require that the order of the Nevanlinna counting function for this curve and for each such a vector is less than order of the curve.

At the end of paper we formulate three unsolved problems concerning asymptotic vectors of entire curve.

Analysis of asymptotic properties of meromorphic functions and relationship with deficiency values play an important role in value distribution theory of meromorphic functions. This topic was first considered in the investigations of F. Iversen [1] and L. Ahlfors [2], etc. Moreover, the fundamental monograph of A. A. Goldbberg and I.V. Ostrovskii [3] contains a whole chapter entitled by "Asymptotic properties of meromorphic functions and deficiencies". They wrote a full review of all investigations on this topic conducted at that time. As for

2010 Mathematics Subject Classification: 30D35.

Keywords: entire curve; Picard exceptional vector; asymptotic vector; meromorphic function; asymptotic value; Picard exceptional value.

doi:10.30970/ms.56.1.48-54

(C) Ya. I. Savchuk, A. I. Bandura, 2021 
the entire curves, the results are more modest (see [4]). In the present paper we will try to close this gap.

We will use main results of theory of entire curves and notation from [4] and [5]. Let us consider transcendental entire curves with linearly independent components and without common zeros.

Let $f: \mathbb{C} \rightarrow \mathbb{C}$ be a meromorphic function. Remind [3, p. 171] that a finite or infinite number $a$ is called an asymptotic value of the meromorphic function $f(z)$, if there exists a continuous curve $L: \mathbb{R}_{+} \rightarrow \mathbb{C}$ given by an equation $z=z(t), 0 \leq t<\infty,|z(t)|<\infty$, $z(t) \rightarrow \infty$ as $t \rightarrow \infty$ such that

$$
\lim _{\substack{z \rightarrow \infty \\ z \in L}} f(z)=\lim _{t \rightarrow \infty} f(z(t))=a .
$$

For $\vec{a}=\left(a_{1}, a_{2}, \ldots, a_{p}\right) \in \mathbb{C}^{p}$ and $\vec{b}=\left(b_{1}, b_{2}, \ldots, b_{p}\right) \in \mathbb{C}^{p}$ the notation $\vec{a} \vec{b}$ means the dot product of these vectors, that is $\vec{a} \vec{b}=\sum_{j=1}^{p} a_{j} \bar{b}_{j}$, where $\bar{b}_{j}$ is complex conjugate to $b_{j}$. Also, we define $\|\vec{a}\|=\sqrt{\left|a_{1}\right|^{2}+\ldots+\left|a_{p}\right|^{2}}$.

We write the meromoprhic function $f(z)$ as a quotient $f(z)=\frac{g_{1}(z)}{g_{2}(z)}$, where $g_{1}$ and $g_{1}$ are entire functions without common zeros. Let us consider an entire curve $\vec{G}(z)=\left(g_{1}(z), g_{2}(z)\right)$ and a vector $\vec{b}_{a}=(1,-\bar{a})$ for finite $a$ and $\vec{b}_{a}=(0 ; 1)$ for $a=\infty$. It is easy to check that

$$
f(z) \rightarrow a \Leftrightarrow \frac{\vec{G}(z) \vec{b}_{a}}{\|\vec{G}(z)\|} \rightarrow 0 .
$$

Let $\vec{G}(z)=\left(g_{1}(z), \ldots, g_{p}(z)\right)$ be an entire transcendental entire curve [4, p. 7] i.e. every component $g_{j}(z)(j \in\{1, \ldots, p\})$ is an entire function and at least one $g_{m}(z) / g_{k}(z)$ is a transcendental meromorphic function $(k \neq m)$.

Taking into account the property (1) we propose the following generalization of concept of asymptotic value for entire curves.

Definition 1. A non-zero vector $\vec{a} \in \mathbb{C}^{p}$ is called an asymptotic vector for the entire curve $\vec{G}: \mathbb{C} \rightarrow \mathbb{C}^{p}$ if there exists a continuous curve $L: \mathbb{R}_{+} \rightarrow \mathbb{C}$ given by an equation $z=z(t)$, $0 \leq t<\infty,|z(t)|<\infty, z(t) \rightarrow \infty$ as $t \rightarrow \infty$ such that

$$
\lim _{\substack{z \rightarrow \infty \\ z \in L}} \frac{\vec{G}(z) \vec{a}}{\|\vec{G}(z)\|}=\lim _{t \rightarrow \infty} \frac{\vec{G}(z(t)) \vec{a}}{\|\vec{G}(z(t))\|}=0 .
$$

The pair $\{\vec{a}, L\}$ is also called an asymptotic spot (see [3, p. 171]). We suppose that asymptotic spots $\left\{\vec{a}_{1}, L_{1}\right\}$ and $\left\{\vec{a}_{2}, L_{2}\right\}$ are equal if

1. $\vec{a}_{1}=\vec{a}_{2}$;

2. there exists a sequence of continuous curves $\gamma_{k}$ such that one end of $\gamma_{k}$ belongs to $L_{1}$, the other end belongs to $L_{2}$, and

$$
\lim _{k \rightarrow \infty} \min _{z \in \gamma_{k}}|z|=\infty, \quad \lim _{\substack{z \rightarrow \cup_{k} \\ z \in \gamma_{k}}} \frac{\vec{G}(z) \vec{a}}{\|\vec{G}(z)\|}=0 .
$$

In [6], there was introduced a concept of Borel exceptional vector for entire curve. A vector $\vec{a} \in \mathbb{C}^{p} \backslash\{\overrightarrow{0}\}$ is called a Borel exceptional vector of entire curve $\vec{G}: \mathbb{C} \rightarrow \mathbb{C}^{p}$, if the growth category of $N(r, \vec{a}, \vec{G})$ is lower than the growth category of $T(r, \vec{G})$. 
A non-zero vector $\vec{a} \in \mathbb{C}^{p}$ is called a Picard exceptional vector of an entire curve $\vec{G}: \mathbb{C} \rightarrow$ $\mathbb{C}^{p}$ if the function $\vec{G}(z) \vec{a}$ has a finite number of zeros in $\{|z|<\infty\}$, i.e. $N(r, \vec{a}, \vec{G})=O(\ln r)$.

In this paper, we will find an answer to the following question: What is a connection between the asymptotic and the exceptional vectors of entire curves in any sense?

Let us remind other necesary notation and concepts from [4], [3] and [5]. For every $p$ dimensional vector $\vec{a}=\left(a_{1}, a_{2}, \ldots, a_{p}\right) \neq \overrightarrow{0}$ the dot product $\vec{G}(z) \vec{a}=\sum_{k=1}^{p} g_{k}(z) \bar{a}_{k}$ is an entire function. Denote by $n(t, \vec{a}, \vec{G})$ a number of zeros of the dot product $\vec{G}(z) \vec{a}$ in the disc $\{z:|z| \leq t\}$, where each zero is counted according to its multiplicity. Every zero of the function $\vec{G}(z) \vec{a}$ is called $a$-point of entire curve $\vec{G}(z)$. Let us denote

$$
N(r, \vec{a}, \vec{G})=\int_{0}^{r} \frac{n(t, \vec{a}, \vec{G})-n(0, \vec{a}, \vec{G})}{t} d t+n(0, \vec{a}, \vec{G}) \ln r
$$

where $n(0, \vec{a}, \vec{G})$ stands for the multiplicity of zero of the dot product $\vec{G}(z) \vec{a}$ at the point $z=0$. The growth characteristic $T(r, \vec{G})$ is defined as following

$$
T(r, \vec{G})=\frac{1}{2 \pi} \int_{0}^{2 \pi} \ln \left\|\vec{G}\left(r e^{i \varphi}\right)\right\| d \varphi .
$$

We will use the definition of the growth category from [3, p.44]. Let $\alpha(r)$ be a function defined for $r>0$, which is non-negative and non-decreasing for sufficiently large $r$ (if $\alpha(r)$ satisfies this condition, we write $\alpha(r) \in \Lambda$ ).

The number $\rho=\rho[\alpha]=\varlimsup_{r \rightarrow+\infty} \frac{\ln ^{+} \alpha(r)}{\ln r}$ is called the order of $\alpha(r)$. The order of the entire curve $\vec{G}: \mathbb{C} \rightarrow \mathbb{C}^{p}$ is defined as the order of the function $T(r, \vec{G})$. The function $\alpha(r)$ is of the type $\sigma$ if $\sigma=\sigma[\alpha]=\overline{\lim }_{r \rightarrow+\infty} \frac{\alpha(r)}{r^{\rho}}$. If $\sigma=0$, we say that $\alpha(r)$ has minimal type; if $0<\sigma<\infty$, we say that $\alpha(r)$ has normal (or mean) type; if $\sigma=\infty$, we say that $\alpha(r)$ has maximal type.

Let $\alpha(r)$ be a function of finite order, i.e. $\rho<+\infty$. We say that $\alpha(r)$ belongs to the convergence class or to the divergence class depending on whether the integral $\int_{1}^{\infty} \frac{\alpha(r)}{r^{\rho+1}} d r$ converges or diverges.

Also, we use a comparison of growth categories for different functions (see a full definition in $[3,6])$. For $\vec{a} \in \mathbb{C}^{p}$ and an entire curve $\vec{G}: \mathbb{C} \rightarrow \mathbb{C}^{p}$ we denote

$$
\delta(\vec{a}, \vec{G})=1-\varlimsup_{r \rightarrow+\infty} \frac{N(r, \vec{a}, \vec{G})}{T(r, \vec{G})} \text { and } \Delta(\vec{a}, \vec{G})=1-\varliminf_{r \rightarrow+\infty} \frac{N(r, \vec{a}, \vec{G})}{T(r, \vec{G})} .
$$

If $\Delta(\vec{a})>0$, then $\vec{a}$ is called a Valiron exceptional vector [12,13], if $\delta(\vec{a})>0$, then $\vec{a}$ is called a Nevanlinna exceptional vector, or deficient vector ( [4, p. 9], see also [5,10]). There is a known concept of averaged deficiency [8]. In addition, there is recent paper [7] on Picard values of $p$-adic meromorphic functions, with investigations of Picard-Hayman behavior of derivatives of meromorphic functions on an algebraically closed field $K$, complete with respect to a non-trivial ultrametric absolute value. More modern bibliography on this topic is listed in review paper of S. Mori [9].

There are many results [3, Ch. 5] on this topic for meromorphic functions. It is known that there is no connection between asymptotic and exceptional values of meromorphic functions. In particular, an asymptotic value does not have to be exceptional in any sense. This fact is easy generalized for a case of entire curves. It is sufficient to consider an entire curve

$$
\vec{G}(z)=\left(1, z, \ldots, z^{p-2}, \frac{\sin z}{z}\right) .
$$

The curve has linearly independent components without common zeros and has the first order. The vector $\vec{a}=(0,0, \ldots 0,1)$ is the asymptotic vector for this curve. It corresponds to 
two different asymptotic spots with asymptotic curves $L_{1}: z(t)=t$ and $L_{2}: z(t)=-t$, but the vector is not Valiron exceptional and hence it is not an exceptional vector in any other sense.

There is a known theorem for meromorphic functions due to F. Iversen which is a result in the opposite direction [3, p. 171]. F. Iversen proved that any Picard exceptional value of meromorphic funtion is an asymptotic value. The following theorem is our modification of Iversen's Theorem in the opposite direction for a case of entire curves.

Theorem 1. Any Picard exceptional vector of transcendental entire curve with linearly independent components and without common zeros is an asymptotic vector.

Proof. Let $\vec{a} \in \mathbb{C}^{p} \backslash\{\overrightarrow{0}\}$ be a Picard exceptional vector for an entire curve

$$
\vec{G}(z)=\left(g_{1}(z), g_{2}(z), \ldots, g_{p}(z)\right) \text {. }
$$

Then the function $\varphi(z)=\vec{G}(z) \vec{a}$ has a finite number of zeros in $\{|z|<\infty\}$. Among the functions $\frac{\varphi(z)}{g_{1}(z)}, \frac{\varphi(z)}{g_{2}(z)}, \ldots, \frac{\varphi(z)}{g_{p}(z)}$ there is at least one transcendental function (for example, it is $\left.\frac{\varphi(z)}{g_{k}(z)}=h(z)\right)$, otherwise all functions $\frac{g_{2}(z)}{g_{1}(z)}, \ldots, \frac{g_{p}(z)}{g_{1}(z)}$ are rational and hence the entire curve $\vec{G}$ is not transcendental (see considerations in [4]). Obviously, every zero of the function $h(z)$ is a zero of the function $\varphi(z)$. Hence, the zero is a Picard exceptional value for $h(z)$. Therefore, there exists a continuous curve $L$ in $\mathbb{C}$ given by equation $z=z(t), 0 \leq t<\infty, z(t) \rightarrow \infty$ as $t \rightarrow \infty$ such that

$$
\lim _{t \rightarrow \infty} h(z(t))=0
$$

Since $\|\vec{G}(z)\|=\sqrt{\left|g_{1}(z)\right|^{2}+\left|g_{2}(z)\right|^{2}+\ldots+\left|g_{p}(z)\right|^{2}} \geq\left|g_{k}(z)\right|$, one has

$$
\frac{|\vec{G}(z) \vec{a}|}{\|\vec{G}(z)\|}=\frac{|\varphi(z)|}{\|\vec{G}(z)\|} \leq \frac{|\varphi(z)|}{\left|g_{k}(z)\right|}=|h(z)| .
$$

Then from (3) we obtain $\lim _{t \rightarrow \infty} \frac{\vec{G}(z(t)) \vec{a}}{\|\vec{G}(z(t))\|}=0$. It means that the vector $\vec{a}$ is asymptotic.

The exceptional vectors in the sense of Borel or Nevanlina and, moreover, in the sense of Valiron do not have to be asymptotic. Below we demonstrate this fact.

In [3, p. 181-183], there was constructed a meromorphic function $f(z)$ of finite positive order, for which $\infty$ is no asymptotic value, but it is the Nevanlinna exceptional value. Let $\psi(z)$ be an entire function whose zeros are poles (counting multiplicities) of the function $f(z)$. Obviously, the functions $(\psi(z))^{p-1}, f(z)(\psi(z))^{p-1},(f(z))^{2}(\psi(z))^{p-1}, \ldots,(f(z) \psi(z))^{p-1}$ are entire and linearly independent functions without common zeros.

Let us consider an entire curve

$$
\vec{G}(z)=\left((\psi(z))^{p-1}, f(z)(\psi(z))^{p-1},(f(z))^{2}(\psi(z))^{p-1}, \ldots,(f(z) \psi(z))^{p-1}\right) .
$$

For this curve we deduce

$$
\begin{gathered}
T(r, \vec{G})=\frac{1}{2 \pi} \int_{0}^{2 \pi} \ln \left\|\vec{G}\left(r e^{i \varphi}\right)\right\| d \varphi= \\
=\frac{1}{2 \pi} \int_{0}^{2 \pi} \ln \left(\left|\psi\left(r e^{i \varphi}\right)\right|^{2 p-2}\left(1+\left|f\left(r e^{i \varphi}\right)\right|^{2}+\ldots+\left|f\left(r e^{i \varphi}\right)\right|^{2 p-2}\right)\right)^{\frac{1}{2}} d \varphi= \\
=\frac{p-1}{2 \pi} \int_{0}^{2 \pi} \ln \left|\psi\left(r e^{i \varphi}\right)\right| d \varphi+\frac{1}{2 \pi} \int_{0}^{2 \pi} \ln \left(1+\left|f\left(r e^{i \varphi}\right)\right|^{2}+\ldots+\left|f\left(r e^{i \varphi}\right)\right|^{2 p-2}\right)^{\frac{1}{2}} d \varphi=
\end{gathered}
$$




$$
\begin{aligned}
& =(p-1) N(r, 0, \psi)+\frac{p-1}{2 \pi} \int_{0}^{2 \pi} \ln ^{+}\left|f\left(r e^{i \varphi}\right)\right| d \varphi+O(1)= \\
= & (p-1)(N(r, f)+m(r, f))+O(1)=(p-1) T(r, f)+O(1) .
\end{aligned}
$$

Above we applied the Jensen formula [3, p. 13, Eq. (4.1)] to the function $\psi$ :

$$
N(r, 1 / g)-N(r, g)=\frac{1}{2 \pi} \int_{0}^{2 \pi} \ln \left|g\left(r e^{i \theta}\right)\right| d \theta-\ln \left|c_{\lambda}\right|,
$$

where $f(z)=c_{\lambda} z^{\lambda}+c_{\lambda} z^{\lambda+1}+\ldots$ be a meromorphic function $\left(c_{\lambda} \neq 0\right)$.

For the vector $\vec{a}=(1,0, \ldots, 0)$ one has $\vec{G}(z) \vec{a}=(\psi(z))^{p-1}$. Hence,

Then

$$
N(r, \vec{a}, \vec{G})=(p-1) N(r, 0, \psi)=(p-1) N(r, f) .
$$

$$
\begin{gathered}
\delta(\vec{a}, \vec{G})=1-\varlimsup_{r \rightarrow \infty} \frac{N(r, \vec{a}, \vec{G})}{T(r, \vec{G})}= \\
=1-\varlimsup_{r \rightarrow \infty} \frac{(p-1) N(r, f)}{(p-1) T(r, f)+O(1)}=1-\varlimsup_{r \rightarrow \infty} \frac{N(r, f)}{T(r, f)}=\delta(\infty, f)>0 .
\end{gathered}
$$

It yields that the vector $\vec{a}=(1,0, \ldots, 0)$ is the Nevanlinna exceptional vector for the entire curve of form (4).

Let us show that the vector $\vec{a}=(1,0, \ldots, 0)$ is not asymptotic for the entire curve. On the contrary, we assume that $\vec{a}=(1,0, \ldots, 0)$ is asymptotic for the curve $\vec{G}$ from (4). Then there exists a continuous curve $L: z=z(t), 0 \leq t<\infty, z(t) \rightarrow \infty$ as $t \rightarrow \infty$ such that (2) is valid. Since

$$
\frac{|\vec{G}(z) \vec{a}|}{\|\vec{G}(z)\|}=\frac{|\psi(z)|^{p-1}}{\left(|\psi(z)|^{2 p-2}\left(1+|f(z)|^{2}+\ldots+|f(z)|^{2 p-2}\right)\right)^{\frac{1}{2}}}=\frac{1}{\sqrt{1+|f(z)|^{2}+\ldots+|f(z)|^{2 p-2}}},
$$

the equality (2) implies $\lim _{t \rightarrow \infty} f(z(t))=\infty$. Thus, $\infty$ is also asymptotic value for the function $f(z)$, but it is a contradiction.

If for any $\rho>1$ as the function $f(z)$ in (4) we choose a function of order $\rho$, for which $\delta(\infty, f)=1$ and the order $N(r, f)$ is less than $\rho$ then $\infty$ is the Borel exceptional value. We obtain an entire curve $\vec{G}(z)$ of order $\rho$, for which and for the vector $\vec{a}=(1,0, \ldots, 0)$ one has $\delta(\vec{a}, \vec{G})=1$ and the order $N(r, \vec{a}, \vec{G})$ is less than $\rho$. However, $\vec{a}=(1,0, \ldots, 0)$ is not asymptotic vector. The following theorem is valid.

Theorem 2. Let $\vec{G}(z)=\left(g_{1}(z), g_{2}(z), \ldots, g_{p}(z)\right)$ be a transcendental entire curve of order $\rho<\infty$ with linearly independent components and without common zeros, the vectors $\vec{a}_{1}, \vec{a}_{2}$, $\ldots, \vec{a}_{p}$ are linearly independent vectors, the orders of $N\left(r, \vec{a}_{1}, \vec{G}\right), N\left(r, \vec{a}_{2}, \vec{G}\right), \ldots, N\left(r, \vec{a}_{p}, \vec{G}\right)$ are less than $\rho$. Then all vectors $\vec{a}_{1}, \vec{a}_{2}, \ldots, \vec{a}_{p}$ are asymptotic vectors.

To prove the theorem we need the following result from [6]:

Lemma 1. ([6, Theorem 3]) Any transcendental entire curve $\vec{G}: \mathbb{C} \rightarrow \mathbb{C}^{p}$ of non-integer or zero order with linearly independent components and without common zeros has at most $(p-1)$ linearly independent Borel exceptional vectors.

Lemma 2. ([3, p. 192, Theorem 2.1.]) Let $f(z)$ be a meromorphic function of order $\rho<\infty$, and orders of $N(r, a)$ and $N(r, b), a \neq b$, be less than $\rho$. Then $a$ and $b$ are asymptotic values. 
Proof of Theorem 2. Without loss of generality we may assume that

$$
\vec{a}_{1}=\vec{e}_{1}=(1,0, \ldots, 0), \vec{a}_{2}=\vec{e}_{2}=(0,1,0, \ldots, 0), \ldots, \vec{a}_{p}=\vec{e}_{p}=(0, \ldots, 0,1) .
$$

Otherwise, as in [6] we can consider an entire curve $\vec{G}_{p}(z)$ with linearly independent components $g_{p_{j}}(z)=\vec{G}(z) \vec{a}_{j}$ without common zeros. Obviously, $\vec{G}_{p}(z) \vec{e}_{j}=\vec{G}(z) \vec{a}_{j}, N\left(r, \vec{e}_{j}, \vec{G}_{p}\right)=$ $N\left(r, \vec{a}_{j}, \vec{G}, T\left(r, \vec{G}_{p}\right)=T(r, \vec{G})+O(1)\right.$.

The vectors $\vec{a}_{1}, \vec{a}_{2}, \ldots, \vec{a}_{p}$ are the Borel exceptional vectors for $\vec{G}(z)$. Then by Lemma 1 the order $\rho$ is natural.

In view of proof of Theorem 3 from [6], for each $k=\{1, \ldots, p\}$ one of the functions $\frac{g_{1}(z)}{g_{k}(z)}, \frac{g_{2}(z)}{g_{k}(z)}, \ldots, \frac{g_{p}(z)}{g_{k}(z)}$ (for example, it is $\left.\frac{g_{m}(z)}{g_{k}(z)}\right)$ necessarily has the order $\rho$. Clearly, the function $\frac{g_{k}(z)}{g_{m}(z)}$ has the same order. Since $g_{j}(z)=\vec{G}(z) \vec{a}_{j}$, one has

$$
N\left(r, 0, \frac{g_{k}}{g_{m}}\right) \leq N\left(r, \vec{a}_{k}, \vec{G}\right), N\left(r, \frac{g_{k}}{g_{m}}\right) \leq N\left(r, \vec{a}_{m}, \vec{G}\right) .
$$

Thus, the orders of $N\left(r, 0, \frac{g_{k}}{g_{m}}\right)$ and $N\left(r, \frac{g_{k}}{g_{m}}\right)$ are less than $\rho$. Then by Lemma 2 the values 0 and $\infty$ are asymptotic values for the function $\frac{g_{k}(z)}{g_{m}(z)}$. From the obvious inequality

$$
\frac{\left|\vec{G}(z) \vec{a}_{k}\right|}{\|\vec{G}(z)\|}=\frac{\left|g_{k}(z)\right|}{\left(\left|g_{1}(z)\right|^{2}+\left|g_{2}(z)\right|^{2}+\ldots+\left|g_{p}(z)\right|^{2}\right)^{\frac{1}{2}}} \leq\left|\frac{g_{k}(z)}{g_{m}(z)}\right|
$$

we obtain that the vector $\vec{a}_{k}$ is asymptotic vector for the entire curve $\vec{G}$.

We do not know an answer to the following question

Problem 1. Is Theorem 2 valid for smaller number of vectors $\vec{a}_{k}$ as $p \geq 3$ ? In other words, is it true or false the next hypothesis

Conjecture 1. Let $\vec{G}(z)=\left(g_{1}(z), g_{2}(z), \ldots, g_{p}(z)\right)$ be a transcendental entire curve of order $\rho<1$ with linearly independent components and without common zeros, $p \geq 3$, the vectors $\vec{a}_{1}, \vec{a}_{2}, \ldots, \vec{a}_{k}$ are linearly independent vectors $(k<p)$, the orders of $N\left(r, \vec{a}_{1}, \vec{G}\right)$, $N\left(r, \vec{a}_{2}, \vec{G}\right), \ldots, N\left(r, \vec{a}_{k}, \vec{G}\right)$ are less than $\rho$. Then all $\vec{a}_{1}, \vec{a}_{2}, \ldots, \vec{a}_{k}$ are asymptotic vectors.

Some interesting problems are arising if we consider relationship between asymptotic vectors of entire curve and its growth. There is known Ahlfors' result [2] on relationship between asymptotic spots of entire function and its growth.

Theorem (A [2]). If an entire function $f(z)$ has $n \geq 2$ asymptotic spots $\left\{\infty, L_{j}\right\}, j \in$ $\{1, \ldots, n\}$, then

$$
\varliminf_{r \rightarrow+\infty} r^{-n / 2} T(r, f)>0 .
$$

This theorem is generalized for meromorphic functions in the following form.

Theorem (B [2]). If $a$ is the Picard exceptional value for meromorphic function $f(z)$ having $n \geq 2$ asymptotic spots $\left\{a, L_{j}\right\}, j \in\{1, \ldots, n\}$, then inequality (6) is valid.

Thus, the following problems seem natural.

Problem 2. Let $\vec{a}$ be the Picard exceptional vector for an entire curve $\vec{G}$ which has $n \geq 2$ asymptotic spots $\left\{\vec{a}, L_{j}\right\}, j \in\{1, \ldots, n\}$. Is it true or false the following inequality

$$
\varliminf_{r \rightarrow+\infty} r^{-n / 2} T(r, f)>0 .
$$


Problem 3. Let $\vec{G}$ be an entire curve with $n$ asymptotic spots $\left\{\vec{a}_{j}, L_{j}\right\}, j \in\{1, \ldots, n\}$. At least one from the vectors $\vec{a}_{1}, \vec{a}_{2}, \ldots, \vec{a}_{n}$ is the Picard exceptional vector for $\vec{G}$. Is it true or false inequality (7)?

Acknowledgments. The research of the second author was funded by the National Research Foundation of Ukraine, 2020.02/0025, 0120U103996.

\section{REFERENCES}

1. F. Iversen, Recherches sur les fonctions inverses des fonctions meromorphes, These, Helsingfors, 1914, $1-67$.

2. L. Ahlfors, Über die asymptotischen Werte der meromorphen Funktionen endlicher Ordnung, Acta Acad. Aboensis. Math. et Phys., 6 (1932), №9, 1-8.

3. A.A. Gol'dberg, I V. Ostrovskii, Value Distribution of Meromorphic Functions, Providence: AMS, 2008. (Translated from Russian ed. Moscow, Nauka, 1970).

4. V.P. Petrenko, Entire curves, Kharkiv: Vyshcha shkola, 1984 (in Russian).

5. Ya.I. Savchuk, Structure of the set of defect vectors of entire and analytic curves of finite order, Ukr. Math. J., 37 (1985), №5, 494-499. doi: 10.1007/BF01061174

6. A.I. Bandura, Ya.I. Savchuk, Structure of the set of Borel exceptional vectors for entire curves, Mat. Stud., 53 (2020), №1, 41-47. doi: 10.30970/ms.53.1.41-47

7. K. Boussaf, Picard values of p-adic meromorphic functions, P-Adic Num. Ultrametr. Anal. Appl., 2 (2010), 285-292. doi: 10.1134/S2070046610040035

8. I.M. Dektyarev, Averaged deficiencies of holomorphic curves and divisors with an excessive deficiency value, Russian Mathematical Surveys, 44 (1989), №1, 237-238. doi: 10.1070/RM1989v044n01 ABEH002015

9. Mori S., Topics on meromorphic mappings and defects, Complex Var. Elliptic Equ., 56 (2011), №1-4, 363-373. doi: 10.1080/17476930903394903

10. Ya.I. Savchuk, Set of deficient vectors of integral curves, Ukr. Math. J., 35 (1983), №3, 334-338. doi: 10.1007/BF01092190

11. Ya.I. Savchuk, Inverse problem of the theory of distribution of the values of entire and analytic curves, J. Soviet Mathematics, 48 (1990), №2, 220-231. doi: 10.1007/BF01095801

12. Ya.I. Savchuk, Valiron deficient vectors of entire curves of finite order, J. Soviet Mathematics, 52 (1990), №5, 3435-3437. doi: 10.1007/BF01099913

13. N. Toda, Holomorphic curves with an infinite number of deficiencies, Proc. Japan Acad. Ser. A Math. Sci., 80 (2004), №6, 90-95. doi: 10.3792/pjaa.80.90.

${ }^{1,2}$ Department of Advanced Mathematics

Ivano-Frankivsk National Technical University of Oil and Gas,

Ivano-Frankivsk, Ukraine

1ysavchukua@gmail.com, ${ }^{2}$ andriykopanytsia@gmail.com

Received 30.03.2021

Revised 12.09.2021 